Note

\section{Concanavalin A-binding Glycoprotein Fraction in the Small Intestine and Feces of Rats}

\author{
Shinobu NaKata and Toshizo KImURA* \\ Laboratory of Food and Nutrition, Department \\ of Home Economics, \\ * Laboratory of Life and Environment, Department of \\ Arts and Sciences, Osaka Kyoiku University, \\ Ikeda, Osaka 563, Japan
}

Received July 19, 1989

In the previous studies, ${ }^{1,2)}$ in which the mechanisms involved in the toxicity of ingested concanavalin A (Con A) were investigated, we observed the following: 1 . The ingested Con A remained unaitered during its passage through the gastrointestinal tract and was rapidly excreted in the faces. 2 . It prevented the adaptive responses of the intestinal sucrase, alkaline phosphatase and leucine aminopeptidase activities, which are localized in the brush border membrane, to dietary nutrients. 3 . The excreted Con $\mathrm{A}$ in the feces was bound to water-insoluble components in the feces, which was suggested by it being released from the feces with an $\alpha$-methyl-D-mannoside solution but not with water. 4 . The water-insoluble components in the feces were confirmed to have an affinity to Con $\mathrm{A}$ in vitro. These findings strongly suggested the presence of a Con A-binding glycoprotein in the small intestine and feces of rats.

The purpose of the present study was to isolate the Con A-binding glycoprotein fraction (CABGP) from the small intestine and feces, and to compare the protein moiety of CABGP isolated from the feces with that isolated from the small intestine.

Male rats of the Wistar strain weighing approximately $100 \mathrm{~g}$ were obtained from JAPAN SLC Inc., Shizuoka, Japan. They were fed on a cereal-based stock diet ad libitum before the start of the experiment. The composition of the experimental diet was as follows: casein, 20 ; sucrose, 70; corn oil, 5; AIN-76 ${ }^{\mathrm{TM}}$ mineral mixture, ${ }^{3)} 4$; vitamin mixture, ${ }^{4)} 1 \%$. The rats were fed on the experimental diet ad libitum for 2 weeks, and were then used for the experiments. Feces excreted during the last week were collected, kept at $4^{\circ} \mathrm{C}$ after being washed with water, and then lyophilized. The stomach, small intestine and cecum were ligated and removed, and their contents were rinsed out with water. Preparation of the jejunum mucosa and the jejunum perfusion were performed in the manners described in the previous studies. ${ }^{5,6)}$ Sodium dodecyl sulfate (SDS) polyacrylamide gel electrophoresis for the protein moieties of CABGP was carried out according to the procedure of Weber and Osborn. ${ }^{7}$ The isolation procedure for CABGP in these tissues and feces was performed by homogenizing 2 grams of the tissues or feces in $100 \mathrm{ml}$ of $1 \%$ sodium deoxycholate (DOC) solution with an Ultra Tarrax homogenizer (Ika Werk, Staufen, West Germany). The homogenized dispersion was stirred at $4^{\circ} \mathrm{C}$ for $20 \mathrm{hr}$, and then centrifuged at $10,000 \times g$ and $5^{\circ} \mathrm{C}$ for $15 \mathrm{~min}$. To the resulting supernatant was added an equal volume of $1.5 \%$ Sanfix 555 (SF, a cationic surface-active agent, Sanyo Chemical Industry, Kyoto, Japan), which combined with DOC and produced an insoluble DOC-SF complex. The suspension was centrifuged at $10,000 \times g$ and $5^{\circ} \mathrm{C}$ for $15 \mathrm{~min}$. The resulting supernatant and an equal volume of $0.1 \mathrm{M}$ phosphate buffer ( $\mathrm{pH} 7.2$ ) were mixed, and then used for the isolation of CABGP. An aliquot of the phosphate-buffered solution was quantitatively applied to a column of a Con A-Sepharose gel (Pharmacia LKB, Uppsala, Sweden), which had been previously equilibrated with $0.1 \mathrm{M}$ phosphate buffer at $\mathrm{pH}$ 7.2. To remove the proteins not bound to the gel, the column was washed with $0.1 \mathrm{M}$ phosphate buffer until there was no absorbance of the eluant at $280 \mathrm{~nm}$. The bound CABGP was then eluted with $0.1 \mathrm{~m}$ phosphate buffer containing $0.5 \mathrm{M} \alpha$-methyl-D-mannoside.

Sucrase-isomaltase complex obtained from the rabbit small intestine is a typical glycoprotein, ${ }^{87}$ sucrase activity in the rat small intestine is localized in the luminal surface (the brush border membrane), ${ }^{9}$ and we have also observed that the sucrase activity fraction isolated from the small intestine had a high affinity to Con A. From these findings, sucrase activity in the small intestine was adopted as a

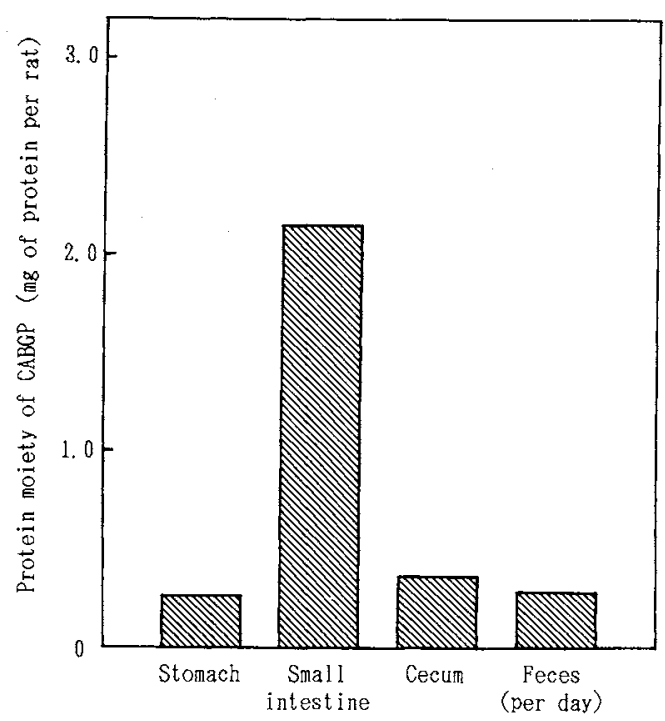

Fig. 1. Distribution of the Concanavalin A-binding Glycoprotein Fraction in the Gastrointestinal Tract and Feces of Rats. 


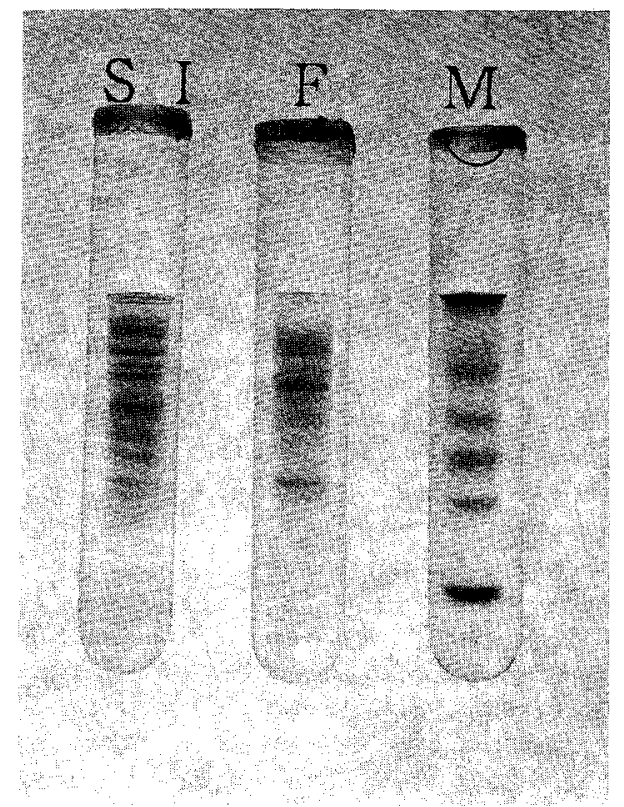

Fig. 2. SDS-Polyacrylamide Gel Electrophoresis of the Concanavalin A-binding Glycoprotein Fractions.

SI, the fraction from the small intestine; $F$, the fraction from the feces; $\mathrm{M}$, cytochrome $c$ as marker proteins (from the top): hexamer, $74.4 \mathrm{~K}$; tetramer, $49.6 \mathrm{~K}$; trimer, $37.2 \mathrm{~K}$; dimer, $24.8 \mathrm{~K}$; monomer, $12.4 \mathrm{~K}$.

criterion for evaluating this CABGP isolation procedure. There was no difference in total sucrase activity between the supernatant of the DOC-solubilized small intestine and that of the SF-treated fraction. The specific activity of sucrase was 2 times greater in the supernatant of the SFtreated fraction, and 40 times in CABGP compared with that in the supernatant of the DOC-solubilized small intestine. These results demonstrate that CABGP solubilized with $D O C$ was not affected by the $S F$ treatment for DOC removal. The distribution of CABGP isolated from the stomach, small intestine, cecum and feces according to this CABGP isolation procedure is shown in Fig. I.
CABGP was detected in all the specimens, but the content in the small intestine was the highest. In SDSpolyacrylamide gel electrophoresis of CABGP isolated from the whole small intestine, the mucosa and the DOCperfusate, there was no difference in the profile of the protein moieties in CABGP among thses fractions. These results suggest that CABGP in the small intestine was localized in the luminal surface. Figure 2 shows the SDSpolyacrylamide electrophoresis of CABGP obtained from the small intestine and feces. There was a definite difference in the relatively small protein moieties between CABGP isolated from the small intestine and that isolated from the feces. CABGP in the feces and that in the small intestine contained $12.5 \%$ and $14.3 \%$ sugars, respectively. The CABGP in the feces, however, might have been a partially digested CABGP derived from that in the small intestine, although no sucrase activity in the fecal CABGP was observed. To verify the suggestion, the relationship between CABGP in the small intestine and that in the feces needs to be extensively investigated, and attempts to find confirmable evidence for the physiological and nutritional significance of CABGP in the feces are in progress in our laboratory.

\section{References}

1) S. Nakata and T. Kimura, J. Nutr., 115, 1621 (1985).

2) S. Nakata and T. Kimura, Agric. Biol. Chem., 50, 645 (1986).

3) American Institute of Nutrition, J. Nutr., 107, 1340 (1977).

4) T. Shizuka, K. Kishi and G. Inoue, J. Nutr. Sci. Vitaminol, 29, 187 (1983).

5) T. Kimura, A. Seto and A. Yoshida, J. Nutr., 108, 1087 (1978).

6) T. Kimura, K. Hasegawa, H. Imamura and A. Yoshida, J. Nutr. Sci. Vitaminol., 29, 153 (1983).

7) K. Weber and M. Osborn, J. Biol. Chem., 244, 4406 (1969).

8) A. Cogoli, H. Mosimann, C. Vock, A. K. Balthazar and G. Semenza, Eur. J. Biochem., 30, 7 (1972).

9) A. Eichholz, Fed. Proc., 28, 30 (1969). 